\title{
Maximal Inequalities for Stochastic Integrals
}

by

\author{
Adam OSĘKOWSKI \\ Presented by Stanisław KWAPIEN
}

Summary. We find the optimal universal constant $C_{p}(1<p \leq \infty)$ in the following inequality. If $X=\left(X_{t}\right)_{t \geq 0}$ is a martingale and $Y=\left(\int_{0}^{t} H_{s} d X_{s}\right)_{t \geq 0}$ for some predictable process $H$ taking values in $[-1,1]$, then

$$
\mathbb{E}\left|\sup _{t \geq 0} Y_{t}\right| \leq C_{p}\|X\|_{p}
$$

1. Introduction. Suppose that $(\Omega, \mathcal{F}, \mathbb{P})$ is a probability space, equipped with a nondecreasing right-continuous family $\left(\mathcal{F}_{t}\right)_{t \geq 0}$ of sub- $\sigma$-fields of $\mathcal{F}$. In addition, assume that $\mathcal{F}_{0}$ contains all the sets of probability 0 . Let $X=\left(X_{t}\right)_{t \geq 0}$ be an adapted real-valued right-continuous martingale with left limits. Let $Y$ be the Itô integral of $H$ with respect to $X$, that is,

$$
Y_{t}=H_{0} X_{0}+\int_{(0, t]} H_{s} d X_{s}, \quad t \geq 0 .
$$

Here $H$ is a predictable process with values in $[-1,1]$. For $p \in[1, \infty]$, let $\|X\|_{p}=\sup _{t \geq 0}\left\|X_{t}\right\|_{p}$. Furthermore, let $X^{*}=\sup _{t \geq 0} X_{t}$ and $|X|^{*}=$ $\sup _{t \geq 0}\left|X_{t}\right|$.

The purpose of this paper is to compare the moments of $X$ and $Y^{*}$. In [B2], Burkholder developed a method to obtain the following sharp estimate.

THEOREM 1.1. If $X$ is a martingale and $Y$ is as above, then

$$
\|Y\|_{1} \leq \gamma\left\||X|^{*}\right\|_{1}
$$

2010 Mathematics Subject Classification: Primary 60G42; Secondary 60G44.

Key words and phrases: martingale, maximal function, stochastic integral, martingale transform, norm inequality. 
where $\gamma=2.536 \ldots$ is the unique solution of the equation

$$
\gamma-3=-\exp \left(\frac{1-\gamma}{2}\right)
$$

The constant is the best possible.

It was shown in [1] that if $X$ is assumed to be a nonnegative supermartingale, then the optimal constant in 11.1 decreases to $2+(3 e)^{-1}=$ $2.1226 \ldots$ The paper [02] contains the following fact.

TheOREM 1.2. If $X$ and $Y$ are as above, then

$$
\left\|Y^{*}\right\|_{1} \leq \beta\left\||X|^{*}\right\|_{1},
$$

where $\beta=2.0856 \ldots$ is the positive solution to the equation

$$
2 \log \left(\frac{8}{3}-\beta_{0}\right)=1-\beta_{0} .
$$

Furthermore, if $X$ is assumed to be nonnegative, then the optimal constant in 1.2 decreases to $14 / 9=1.5555 \ldots$

In the present paper we continue this line of research and provide new sharp bounds for the first moment of $Y^{*}$ by $\|X\|_{p}$ for $p>1$. If $p=1$, then there is no finite constant $C_{1}$ such that $\left\|Y^{*}\right\|_{1} \leq C_{1}\|X\|_{1}$, even when $Y=X$. For example, take $X_{t}=e^{\alpha W_{t}-\alpha^{2} t / 2}$, where $W$ is the Wiener process; then $\mathbb{E} X^{*}=\infty$ and $\mathbb{E}\left|X_{t}\right|=\mathbb{E} X_{t}=1$ for all $t$. Let

$$
C_{p}= \begin{cases}\Gamma\left(\frac{2 p-1}{p-1}\right)^{1-1 / p} & \text { if } 1<p \leq 2, \\ \left(2^{p /(p-1)}-\frac{p}{p-1} \int_{1}^{2} s^{1 /(p-1)} e^{s-2} d s\right)^{1-1 / p} & \text { if } 2<p<\infty \\ 1+e^{-1} & \text { if } p=\infty .\end{cases}
$$

Here is our main result.

TheOrem 1.3. Suppose $X$ is a martingale and $Y$ is as above. If $1<$ $p \leq \infty$, then

$$
\left\|Y^{*}\right\|_{1} \leq C_{p}\|X\|_{p} .
$$

The constant $C_{p}$ is the best possible.

By the approximation arguments of Bichteler [Bi], the theorem above is a quick consequence of its discrete-time version, which we will prove next. Suppose that $(\Omega, \mathcal{F}, \mathbb{P})$ is a probability space, filtered by $\left(\mathcal{F}_{n}\right)_{n \geq 0}$. Let $f=$ $\left(f_{n}\right)_{n \geq 0}$ be an adapted martingale and $g=\left(g_{n}\right)_{n \geq 0}$ be its transform by a predictable sequence $v=\left(v_{n}\right)_{n \geq 0}$ bounded in absolute value by 1 . That is, 
we have

$$
g_{n}=\sum_{k=0}^{n} v_{k} d f_{k}, \quad n=0,1,2, \ldots,
$$

where $d f_{0}=f_{0}$ and $d f_{k}=f_{k}-f_{k-1}$ for $k \geq 1$. Here by predictability of $v$ we mean that $v_{0}$ is $\mathcal{F}_{0}$-measurable and for any $k \geq 1, v_{k}$ is measurable with respect to $\mathcal{F}_{k-1}$. In the particular case when each $v_{k}$ is deterministic and takes values in the set $\{-1,1\}$, we will say that $g$ is a \pm 1 transform of $f$.

Denote $f_{n}^{*}=\max _{k \leq n} f_{k}$ and $f^{*}=\sup _{k} f_{k}$.

THEOREM 1.4. Suppose $f, g$ are martingales such that $g$ is a transform of $f$ by a predictable sequence bounded in absolute value by 1 . If $1<p \leq \infty$, then

$$
\left\|g^{*}\right\|_{1} \leq C_{p}\|f\|_{p}
$$

A few words about the organization of the paper. The proof of our result is based on Burkholder's technique, which exploits properties of certain special functions; the method is described in the next section. Section 3 contains the proof of (1.3) and (1.4) for $p \in(1,2]$, while the case $p \in(2, \infty]$ is postponed to the final part of the paper, Section 4.

2. Some reductions and the method of proof. Using approximation arguments of Bichteler [Bi], it suffices to focus on the discrete-time setting. Now, with no loss of generality, we may assume that in (1.4) we deal with simple sequences $f$ and $g$. By simplicity of $f$ we mean that for any integer $n$, the random variable $f_{n}$ takes only a finite number of values and there exists a deterministic number $N$ such that $f_{N}=f_{N+1}=\cdots$ with probability 1 . Clearly, if $f$ and $g$ are simple, then the almost sure limits $f_{\infty}$ and $g_{\infty}$ exist and are finite. Next, we may assume that $g_{0} \geq 0$ almost surely, which gives $\left|g^{*}\right|=g^{*}$. Indeed, it suffices to replace $v_{0}$ by $\operatorname{sgn} f_{0}$ if necessary; then $\left|g^{*}\right|$ increases, so we obtain a stronger estimate to prove.

The key reduction is that it suffices to work with \pm 1 transforms only. Recall Lemma A.1 from B1.

LEMMA 2.1. Let $g$ be the transform of a martingale $f$ by a real-valued predictable sequence $v$ uniformly bounded in absolute value by 1 . Then for each $j \geq 1$ there exist martingales $F^{j}=\left(F_{n}^{j}\right)_{n \geq 0}$ and $G^{j}=\left(G_{n}^{j}\right)_{n \geq 0}$ such that for $j \geq 1$ and $n \geq 0$,

$$
f_{n}=F_{2 n+1}^{j} \quad \text { and } \quad g_{n}=\sum_{j=1}^{\infty} 2^{-j} G_{2 n+1}^{j},
$$

and $G^{j}$ is a \pm 1 transform of $F^{j}$.

To see how the lemma works in our setting, suppose we have established (1.4) for \pm 1 transforms. Now, if $g$ is a transform of $f$, then Lemma 2.1 
gives us the processes $F^{j}$ and $G^{j}$, for which we may write

$$
\begin{aligned}
\left\|g^{*}\right\|_{1} & =\left\|\sup _{n} \sum_{j=1}^{\infty} 2^{-j} G_{2 n+1}^{j}\right\|_{1} \leq \sum_{j=1}^{\infty} 2^{-j}\left\|G^{j *}\right\|_{1} \\
& \leq C_{p} \sum_{j=1}^{\infty} 2^{-j}\left\|F^{j}\right\|_{p}=C_{p}\|f\|_{p},
\end{aligned}
$$

as needed.

Observe that in the proof of 1.4 we may assume that $p$ is finite. Let $\mathcal{A}=\left\{(x, y, z) \in \mathbb{R}^{3}: y \leq z\right\}$ and define $V_{p}: \mathcal{A} \rightarrow \mathbb{R}$ by

$$
V_{p}(x, y, z)= \begin{cases}y \vee z-|x|^{p}+\gamma_{p}(0) & \text { if } 1<p \leq 2, \\ y \vee z-|x|^{p}+M_{p} & \text { if } 2<p<\infty\end{cases}
$$

where $\gamma_{p}$ is given by (3.1) and $M_{p}$ is introduced in (4.2) below. It is enough to show that

$$
\mathbb{E} V_{p}\left(f_{\infty}, g_{\infty}, g_{\infty}^{*}\right) \leq 0
$$

for all simple martingales $f, g$ such that $g$ is a \pm 1 transform of $f$. This follows from a standard homogenization procedure. Indeed: for $1<p \leq 2$, apply (2.1) to the martingales $f / \lambda, g / \lambda$, where $\lambda>0$ is fixed. This yields

$$
\mathbb{E} g_{\infty}^{*} \leq \lambda^{1-p} \mathbb{E}\left|f_{\infty}\right|^{p}-\lambda \gamma_{p}(0) \text {. }
$$

Now the choice

$$
\lambda=\left(-\frac{p-1}{\gamma_{p}(0)}\right)^{1 / p}\|f\|_{p}
$$

gives (1.4). For $p>2$ the reasoning is the same.

The estimate (2.1) will be achieved if we find a function $U: \mathcal{A} \rightarrow \mathbb{R}$ with the following three properties.

$1^{\circ}$ For any $\varepsilon \in\{-1,1\}$ and $(x, y, z) \in \mathcal{A}$ there is a number $c=c(\varepsilon, x, y, z)$ such that for all $d \in \mathbb{R}$,

$$
U(x+\varepsilon d, y+d,(y+d) \vee z) \leq U(x, y, z)+c d .
$$

$2^{\circ} U(x, y, z) \geq V_{p}(x, y, z)$ for all $(x, y, z)$.

$3^{\circ} U(x, y, y) \leq 0$ for all $x, y$ such that $x=|y|$.

The class of all functions $U$ satisfying $1^{\circ}-3^{\circ}$ will be denoted by $\mathcal{U}\left(V_{p}\right)$.

Sometimes it is convenient to replace $1^{\circ}$ with the following equivalent condition (see [B2]):

$1^{\text {of }}$ For any $\varepsilon \in\{-1,1\},(x, y, z) \in \mathcal{A}$ and any simple centered random variable $T$, we have

$$
\mathbb{E} U(x+\varepsilon T, y+T,(y+T) \vee z) \leq U(x, y, z) .
$$


The relation between the inequality 2.1 and the class $\mathcal{U}\left(V_{p}\right)$ is described in the following fact.

THEOREM 2.2. If the class $\mathcal{U}\left(V_{p}\right)$ is nonempty, then the inequality 2.1 holds for any simple $f, g$ such that $g$ is a \pm 1 transform of $f$.

Proof. Take $U \in \mathcal{U}\left(V_{p}\right)$ and simple $f, g$ such that $g$ is a \pm 1 transform of $f$. The process $\left(U\left(f_{n}, g_{n}, g_{n}^{*}\right)\right)_{n \geq 0}$ is a supermartingale: indeed, the inequality $\mathbb{E}\left[U\left(f_{n}, g_{n}, g_{n}^{*}\right) \mid \mathcal{F}_{n-1}\right] \leq \bar{U}\left(f_{n-1}, g_{n-1}, g_{n-1}^{*}\right), n \geq 1$, follows from the conditional form of $1^{\circ \prime}$, with $x=f_{n-1}, y=g_{n-1}, z=g_{n-1}^{*}, T=d g_{n}$ and $\varepsilon \in\{-1,1\}$ such that $d g_{n}=\varepsilon d f_{n}$. Consequently, using $2^{\circ}$ and then $3^{\circ}$, one gets

$$
\mathbb{E} V_{p}\left(f_{\infty}, g_{\infty}, g_{\infty}^{*}\right) \leq \mathbb{E} U\left(f_{\infty}, g_{\infty}, g_{\infty}^{*}\right) \leq \mathbb{E} U\left(f_{0}, g_{0}, g_{0}^{*}\right) \leq 0
$$

Thus the problem of proving a given martingale inequality 2.1 is reduced to the problem of constructing a function with properties $1^{\circ}, 2^{\circ}$ and $3^{\circ}$.

It turns out that the implication can be reversed. For $V_{p}$ as above, consider $U_{0}: \mathcal{A} \rightarrow \mathbb{R}$ given by

$$
U_{0}(x, y, z)=\sup \mathbb{E} V_{p}\left(f_{\infty}, g_{\infty}, g_{\infty}^{*} \vee z\right)
$$

where the supremum is taken over the class $M(x, y)$ of all pairs $(f, g)$ of simple martingales such that $\left(f_{0}, g_{0}\right)=(x, y)$ and $d g_{n}= \pm d f_{n}$ for all $n \geq 1$ (that is, there is a deterministic $v=\left(v_{n}\right)_{n \geq 1}$ taking values in $\{-1,1\}$ such that $\left.d g_{n}=v_{n} d f_{n}, n \geq 1\right)$.

THEOREM 2.3. If 2.1 is valid, then the class $\mathcal{U}\left(V_{p}\right)$ is nonempty and $U_{0}$ is its least element.

For the proof, one needs to slightly modify the argument used in B2 (see Theorem 2.2 there). Theorem 2.3 will be quite useful in the proof of the optimality of the constants $C_{p}$. In the next two sections we will construct appropriate special functions.

3. The proof of 1.4 for $1<p \leq 2$. We start by defining a function $\gamma_{p}:[0, \infty) \rightarrow(-\infty, 0]$ by

$$
\gamma_{p}(t)=-\exp \left(p t^{p-1}\right) \int_{t}^{\infty} \exp \left(-p s^{p-1}\right) d s .
$$

Since

$$
\gamma_{p}(t)=-\int_{0}^{\infty} \exp \left\{-p(p-1) \int_{0}^{s}(t+u)^{p-2} d u\right\} d s
$$

the function $\gamma_{p}$ is nonincreasing on $[0, \infty)$. Let $G_{p}:\left(-\infty, \gamma_{p}(0)\right] \rightarrow[0, \infty)$ denote the inverse of the function $t \mapsto \gamma_{p}(t)-t, t \geq 0$. We will need the following estimate. 
Lemma 3.1. We have $G_{p} G_{p}^{\prime \prime}+(p-2)\left(G_{p}^{\prime}\right)^{2} \leq 0$.

Proof. The inequality to be proved is equivalent to $\left(G_{p} / G_{p}^{\prime}\right)^{\prime} \geq p-1$. Since $\gamma_{p}^{\prime}(t)=p(p-1) t^{p-2} \gamma_{p}(t)+1$, we obtain

$$
G_{p}^{\prime}(x)=\left(\gamma_{p}\left(G_{p}(x)\right)-1\right)^{-1}=\left[p(p-1) G_{p}^{p-2}(x)\left(x+G_{p}(x)\right)\right]^{-1}
$$

and

$$
1+G_{p}^{\prime}(x)=\frac{\gamma_{p}\left(G_{p}(x)\right)}{p(p-1) G_{p}^{p-2}(x) \gamma_{p}\left(G_{p}(x)\right)} .
$$

Therefore

$$
\left(\frac{G_{p}(x)}{G_{p}^{\prime}(x)}\right)^{\prime}=\left[p(p-1) G_{p}^{p-1}(x)\left(x+G_{p}(x)\right)\right]^{\prime}=p-1+\frac{G_{p}(x) \gamma_{p}^{\prime}\left(G_{p}(x)\right)}{\gamma_{p}(G(x))} \geq p-1,
$$

because $G_{p}(x) \geq 0$ and $\gamma_{p}\left(G_{p}(x)\right)<0, \gamma_{p}^{\prime}\left(G_{p}(x)\right) \leq 0$.

Now we are ready to introduce a special function. Let

$$
\begin{aligned}
& D_{1}=\left\{(x, y, z) \in \mathcal{A}: y-z-|x| \geq \gamma_{p}(0)\right\}, \\
& D_{2}=\left\{(x, y, z) \in \mathcal{A}: y-z-|x|<\gamma_{p}(0) \text { and }|x| \geq G_{p}(y-z-|x|)\right\}, \\
& D_{0}=\mathcal{A} \backslash\left(D_{1} \cup D_{2}\right) .
\end{aligned}
$$

Let $U_{p}: \mathcal{A} \rightarrow \mathbb{R}$ be given by

$$
U_{p}(x, y, z)= \begin{cases}-\frac{(y-z)^{2}-x^{2}}{2 \gamma_{p}(0)}+\frac{\gamma_{p}(0)}{2}+y & \text { on } D_{1}, \\ z+\gamma_{p}(0)+(p-1) G_{p}(y-z-|x|)^{p} & \\ -p|x| G_{p}(y-z-|x|)^{p-1} & \text { on } D_{2}, \\ z-|x|^{p}+\gamma_{p}(0) & \text { on } D_{0} .\end{cases}
$$

We will now verify that $U_{p}$ belongs to $\mathcal{U}\left(V_{p}\right)$ and thus establish 1.4. To do this, it suffices to show the following fact.

LEMMA 3.2.

(i) The function $U_{p}$ is of class $C^{1}$ in the interior of $\mathcal{A}$.

(ii) For any $\varepsilon \in\{-1,1\}$ and $(x, y, z) \in \mathcal{A}$, the function $F=F_{\varepsilon, x, y, z}$ : $(-\infty, z-y] \rightarrow \mathbb{R}$, given by $F(t)=U_{p}(x+\varepsilon t, y+t, z)$, is concave.

(iii) For any $\varepsilon \in\{-1,1\}$ and $x, y, h \in \mathbb{R}$,

$$
U_{p}(x+\varepsilon t, y+t,(y+t) \vee y) \leq U_{p}(x, y, y)+\varepsilon U_{p x}(x, y, y) t+t .
$$

(iv) We have

(v) We have

$$
U_{p}(x, y, z) \geq V_{p}(x, y, z) \quad \text { for }(x, y, z) \in \mathcal{A} .
$$

$$
\sup U_{p}(x, y, y)=0
$$

where the supremum is taken over all $x, y$ satisfying $|x|=|y|$. 
Proof. (i) This is straightforward: $U_{p}$ is of class $C^{1}$ in the interior of $D_{0}, D_{1}$ and $D_{2}$, so the claim reduces to tedious verification that the partial derivatives $U_{p x}, U_{p y}$ and $U_{p z}$ match at the common boundaries of $D_{0}, D_{1}$ and $D_{2}$.

(ii) In view of (i), it suffices to show that $F^{\prime \prime}(t) \leq 0$ for those $t$ for which the second derivative exists. In view of the translation property $F_{\varepsilon, x, y, z}(u)=$ $F_{\varepsilon, x+\varepsilon s, y+s, z}(u-s)$, valid for all $u$ and $s$, it suffices to check $F^{\prime \prime}(t) \leq 0$ only for $t=0$. Furthermore, since we have $U_{p x}(0, y, z)=0$ and $U_{p}(x, y, z)=$ $U_{p}(-x, y, z)$, we may restrict ourselves to $x>0$.

If $\varepsilon=1$, then we easily verify that $F^{\prime \prime}(0)=0$ if $(x, y, z)$ lies in the interior $\left(D_{1} \cup D_{2}\right)^{o}$ of $D_{1} \cup D_{2}$ and $F^{\prime \prime}(0)=-p(p-1) x^{p-2} \leq 0$ if $(x, y, z) \in D_{0}^{o}$. Thus it remains to check the case $\varepsilon=-1$. We start from the observation that $F^{\prime \prime}(0)=0$ if $(x, y, z) \in D_{1}^{o}$. If $(x, y, z) \in D_{2}^{o}$, then

$$
F^{\prime \prime}(0)=4 p(p-1) G_{p}^{p-3}\left[G_{p} G_{p}^{\prime}\left(G_{p}^{\prime}+1\right)+\left(G_{p}-x\right)\left((p-2)\left(G_{p}^{\prime}\right)^{2}+G_{p} G_{p}^{\prime \prime}\right)\right]
$$

where all the functions on the right are evaluated at $x_{0}=y-z-x$. Since $y \leq z$, we have $x \leq-x_{0}$ and, in view of Lemma 3.1.

$$
\begin{aligned}
F^{\prime \prime}(0) \leq & 4 p(p-1) G_{p}^{p-3}\left(x_{0}\right)\left[G_{p}\left(x_{0}\right) G_{p}^{\prime}\left(x_{0}\right)\left(G_{p}^{\prime}\left(x_{0}\right)+1\right)\right. \\
& \left.+\left(G_{p}\left(x_{0}\right)+x_{0}\right)\left((p-2)\left(G_{p}^{\prime}\left(x_{0}\right)\right)^{2}+G_{p}\left(x_{0}\right) G_{p}^{\prime \prime}\left(x_{0}\right)\right)\right] \\
= & 0 .
\end{aligned}
$$

Here in the last step we have used the equality

$$
G_{p}(x) G_{p}^{\prime \prime}(x)+(p-2)\left(G_{p}^{\prime}(x)\right)^{2}=-\frac{G_{p}(x) G_{p}^{\prime}(x)\left(G_{p}^{\prime}(x)+1\right)}{G_{p}(x)+x}
$$

which can be easily extracted from the proof of Lemma 3.1. Thus we are done with $D_{2}^{o}$. Finally, if $(x, y, z)$ belongs to the interior of $D_{0}$, then $F^{\prime \prime}(0)=$ $-p(p-1) x^{p-2} \leq 0$.

(iii) We may assume that $x \geq 0$, due to the symmetry of the function $U_{p}$. Note that $U_{p y}(x, y-, y)=1$; therefore, if $t \leq 0$, then the estimate follows from the concavity of $U_{p}$ along the lines of slope \pm 1 , established in the previous part. If $t>0$, then

$$
U_{p}(x+\varepsilon t, y+t,(y+t) \vee y)=U_{p}(x, y+t, y+t)=y+t+U_{p}(x+\varepsilon t, 0,0)
$$

and hence we will be done if we show that the function $s \mapsto U_{p}(s, 0,0)$ is concave on $[0, \infty)$. However, its second derivative equals $1 / \gamma_{p}(0)<0$ for $s<\gamma_{p}(0)$ and

$$
\begin{aligned}
p(p-1) G_{p}^{p-3}(-s) & {\left[\left(G_{p}(-s)-s\right)\left((p-2)\left(G_{p}^{\prime}(-s)\right)^{2}+G_{p}(-s)^{p-2} G_{p}^{\prime \prime}(-s)\right)\right.} \\
& \left.+G_{p}(-s) G_{p}^{\prime}(-s)\left(G_{p}^{\prime}(-s)+2\right)\right] \\
= & p(p-1) G_{p}(-s)^{p-2} G_{p}^{\prime}(-s) \leq 0
\end{aligned}
$$

for $s>\gamma_{p}(0)$. Here we have used the equality from 3.5 , with $x_{0}=-s$. 
(iv) Again, it suffices to deal only with nonnegative $x$. On the set $D_{0}$ both sides of (3.3) are equal. To prove the majorization on $D_{2}$, let $\Phi(s)=\gamma_{p}(0)-s^{p}$ for $s \geq 0$. Observe that

$$
\begin{aligned}
U_{p}(x, y, z)= & z+\Phi\left(G_{p}(y-z-x)\right) \\
& +\Phi^{\prime}\left(G_{p}(y-z-x)\right)\left(x-G_{p}(y-z-x)\right),
\end{aligned}
$$

which, by concavity of $\Phi$, is not smaller than $z+\Phi(x)$. Finally, the estimate for $(x, y, z) \in D_{1}$ is a consequence of the fact that

so

$$
U_{p y}(x, y-, z)=\frac{\gamma_{p}(0)-(y-z)}{\gamma_{p}(0)} \geq 0,
$$

$$
U_{p}(x, y, z)-V_{p}(x, y, z) \geq U_{p}\left(x, y_{0}, z\right)-V_{p}\left(x, y_{0}, z\right) \geq 0 .
$$

Here $\left(x, y_{0}, z\right) \in \partial D_{2}$ and the latter bound follows from the majorization on $D_{2}$, which we have just established.

(v) We have

$$
U_{p}(x, y, y)=U_{p}(|x|, 0,0)+y \leq U_{p}(|x|, 0,0)+|x| .
$$

As shown in the proof of (iii), $s \mapsto U_{p}(s, 0,0), s \geq 0$, is concave, hence so is the function $s \mapsto U_{p}(s, 0,0)+s, s \geq 0$. It suffices to note that its derivative vanishes at $-\gamma_{p}(0)$, so the value at this point (which is equal to 0 ) is the supremum we are searching for.

Sharpness. As shown by Peskir $[\mathrm{P}]$, the Doob-type bound

$$
\left\|B_{\tau}^{*}\right\|_{1} \leq \Gamma\left(\frac{2 p-1}{p-1}\right)^{1-1 / p}\left\|B_{\tau}\right\|_{p}, \quad 1<p \leq 2,
$$

is sharp. Here $B$ is a Brownian motion (not necessarily starting from 0 ) and $\tau$ is a stopping time for $B$ satisfying $\tau \in L^{p / 2}$. Consequently, the estimate (1.4) is also sharp, even if $X=Y$.

4. The proof of (1.4) for $p>2$. Suppose that $p$ is finite. Let $\gamma_{p}$ : $[0, \infty) \rightarrow(-\infty, 0)$ be given by

$$
\begin{aligned}
\gamma_{p}(t) & =\exp \left(-p t^{p-1}\right)\left[-\int_{p^{-1 /(p-1)}}^{t} \exp \left(p s^{p-1}\right) d s-p^{-1 /(p-1)} e\right] \\
& =-t+p(p-1) \exp \left(-p t^{p-1}\right) \int_{p^{-1 /(p-1)}}^{t} s^{p-1} \exp \left(p s^{p-1}\right) d s
\end{aligned}
$$

if $t>p^{-1 /(p-1)}$, and

$$
\gamma_{p}(t)=(p-2)\left(t-p^{-1 /(p-1)}\right)-p^{-1 /(p-1)}
$$

if $t \in\left[0, p^{-1 /(p-1)}\right]$. We start with the following straightforward fact. 
LEMMA 4.1. The function $\gamma_{p}$ is of class $C^{1}$ and nondecreasing.

Proof. The first assertion can be verified easily. To prove the second one, note that it suffices to show $\gamma_{p}^{\prime}(t) \geq 0$ for $t \geq p^{-1 /(p-1)}$. Equivalently, $\gamma_{p}^{\prime}(t) \geq 0$ reads

$$
t^{2-p} \exp \left(p t^{p-1}\right)-p(p-1) \int_{p^{-1 /(p-1)}}^{t} \exp \left(p s^{p-1}\right) d s-p^{(p-2) /(p-1)}(p-1) e \leq 0 .
$$

However, the inequality is true for $t=p^{-1 /(p-1)}$ and the derivative of the left-hand side equals $(2-p) t^{1-p} \exp \left(p t^{p-1}\right) \leq 0$. This completes the proof.

Let $G_{p}:[0, \infty) \rightarrow\left[p^{-1 /(p-1)}, \infty\right)$ be the inverse to the function $t \mapsto$ $\gamma_{p}(t)+t, t \geq p^{-1 /(p-1)}$ (the function is invertible, by the previous fact). We have the following version of Lemma 3.1 .

LEMmA 4.2. We have $G_{p} G_{p}^{\prime \prime}+(p-2)\left(G_{p}^{\prime}\right)^{2} \geq 0$.

Proof. It can be verified that

$$
G_{p}(x) G_{p}^{\prime \prime}(x)+(p-2)\left(G_{p}^{\prime}(x)\right)^{2}=\frac{G_{p}(x) G_{p}^{\prime}(x)\left(G_{p}^{\prime}(x)-1\right)}{x-G_{p}(x)},
$$

and this is nonnegative: it follows from the very definition of $G_{p}$ that $G_{p}(x) \geq 0, G_{p}^{\prime}(x) \geq 0$ and $G_{p}^{\prime}(x) \leq 1, x-G_{p}(x)<0$.

Define

$$
M_{p}=\frac{p-1}{p^{p /(p-1)}}\left[2^{p /(p-1)}-\frac{p}{p-1} \int_{1}^{2} s^{1 /(p-1)} e^{s-2} d s\right] .
$$

Let $H_{p}: \mathbb{R}^{2} \rightarrow \mathbb{R}$ be given by

$$
H_{p}(x, y)=(p-1)^{1-p}(-(p-1)|x|+|y|)(|x|+|y|)^{p-1}
$$

and put

$$
\begin{aligned}
& D_{1}=\left\{(x, y, z) \in \mathcal{A}: y-z \geq \gamma_{p}(x), x+y-z \leq 0\right\}, \\
& D_{2}=\left\{(x, y, z) \in \mathcal{A}: y-z \geq \gamma_{p}(x), x+y-z>0\right\}, \\
& D_{0}=\mathcal{A} \backslash\left(D_{1} \cup D_{2}\right) .
\end{aligned}
$$

Introduce $U_{p}: \mathcal{A} \rightarrow \mathbb{R}$ by

$$
U_{p}(x, y, z)= \begin{cases}z+H_{p}\left(x, y-z+(p-1) p^{-1 /(p-1)}\right)-M_{p} & \text { on } D_{1}, \\ z-M_{p}+(p-1) G_{p}(|x|+y-z)^{p} & \text { on } D_{2} \\ -p|x| G_{p}(|x|+y-z)^{p-1} & \text { on } D_{0} .\end{cases}
$$

Here is the analogue of Lemma 3.2. Again, once we show it, we will be done with the proof of 1.4 . 
LEMMA 4.3.

(i) The function $U_{p}$ is of class $C^{1}$.

(ii) For any $\varepsilon \in\{-1,1\}$ and $(x, y, z) \in \mathcal{A}$, the function $F=F_{\varepsilon, x, y, z}$ : $(-\infty, z-y] \rightarrow \mathbb{R}$, given by $F(t)=U_{p}(x+\varepsilon t, y+t, z)$, is concave.

(iii) For any $\varepsilon \in\{-1,1\}$ and $x, y, h \in \mathbb{R}$,

$$
U_{p}(x+\varepsilon t, y+t,(y+t) \vee y) \leq U_{p}(x, y, y)+\varepsilon U_{p x}(x, y, y) t+t .
$$

(iv) We have

$$
U_{p}(x, y, z) \geq V_{p}(x, y, z) \quad \text { for }(x, y, z) \in \mathcal{A} .
$$

(v) We have

$$
\sup U_{p}(x, y, y)=0
$$

where the supremum is taken over all $x, y$ satisfying $|x|=|y|$.

Proof. (i) Straightforward.

(ii) We proceed as in the proof of Lemma 3.2(ii) and check $F^{\prime \prime}(0) \leq 0$ for $x>0$ and $(x, y, z)$ lying in the interior of some $D_{i}$.

If $\varepsilon=1$, there is nothing to check: we have $F^{\prime \prime}(0)=0$ if $(x, y, z) \in$ $\left(D_{1} \cup D_{2}\right)^{o}$ or $F^{\prime \prime}(0)=-p(p-1) x^{p-2} \leq 0$ if $(x, y, z) \in D_{0}^{o}$. It remains to verify the case $\varepsilon=-1$. If $(x, y, z)$ belongs to the interior of $D_{1}$, then $F^{\prime \prime}(0) \leq 0$; this follows from the fact that for any $\left(x^{\prime}, y^{\prime}\right) \in \mathbb{R}^{2}$, the function $t \mapsto H_{p}\left(x^{\prime}+t, y^{\prime}-t\right)$ is concave (see [B1, p. 17]). If $(x, y, z) \in D_{2}^{o}$, then

$$
F^{\prime \prime}(0)=4 p(p-1) G_{p}^{p-3}\left[G_{p} G_{p}^{\prime}\left(G_{p}^{\prime}-1\right)+\left(G_{p}-x\right)\left((p-2)\left(G_{p}^{\prime}\right)^{2}+G_{p} G_{p}^{\prime \prime}\right)\right],
$$

where all the functions on the right are evaluated at $x_{0}=x+y-z$. We have $y \leq z$, so $x \leq x_{0}$ and, by Lemma 4.2 .

$$
\begin{aligned}
F^{\prime \prime}(0) \leq & 4 p(p-1) G_{p}^{p-3}\left(x_{0}\right)\left[G_{p}\left(x_{0}\right) G_{p}^{\prime}\left(x_{0}\right)\left(G_{p}^{\prime}\left(x_{0}\right)-1\right)\right. \\
& \left.+\left(G_{p}\left(x_{0}\right)-x_{0}\right)\left((p-2)\left(G_{p}^{\prime}\left(x_{0}\right)\right)^{2}+G_{p}\left(x_{0}\right) G_{p}^{\prime \prime}\left(x_{0}\right)\right)\right] \\
= & 0,
\end{aligned}
$$

where we have used the equality from (4.1). Finally, if $(x, y, z)$ belongs to the interior of $D_{0}$, then $F^{\prime \prime}(0)=-p(p-1) x^{p-2} \leq 0$.

(iii) We have $U_{p y}(x, y-, y)=1$ and $U_{p}(x, y, y)=y+U_{p}(x, 0,0)$. Therefore, arguing as in the proof of Lemma 3.2, we see that it suffices to show that the function $s \mapsto U_{p}(s, 0,0), s>0$, is concave. Indeed, its second derivative at $s$ equals

$$
-p(p-1) G_{p}^{p-2}(s) G_{p}^{\prime}(s) \leq 0
$$

and we are done.

(iv) The majorization can be proved in the same manner as in Lemma 3.2 , using the concave function $\Phi(s)=-s^{p}, s \geq 0$. The details are left to the reader. 
(v) Observe that

$$
U_{p}(x, y, y)=y+U_{p}(|x|, 0,0) \leq|x|+U_{p}(|x|, 0,0)
$$

Denoting the right-hand side by $\Psi(|x|)$, we find that $\Psi$ is concave on $(0, \infty)$ (see the proof of (iii)) and

$$
\begin{aligned}
\Psi^{\prime}(t) & =p(p-1) G_{p}^{\prime}(t) G_{p}(t)^{p-2}\left(G_{p}(t)-t\right)-p G_{p}(t)^{p-1}+1 \\
& =-p G_{p}(t)^{p-1}+2 .
\end{aligned}
$$

Consequently, $\Psi$ attains its maximum at the point $t_{0}$ satisfying $G_{p}\left(t_{0}\right)=$ $(2 / p)^{1 /(p-1)}$, or

$$
\begin{aligned}
t_{0} & =\gamma_{p}\left((2 / p)^{1 /(p-1)}\right)+(2 / p)^{1 /(p-1)} \\
& =p(p-1) e^{-2} \int_{p^{-1 /(p-1)}}^{(p / 2)^{-1 /(p-1)}} s^{p-1} \exp \left(p s^{p-1}\right) d s \\
& =p^{-1 /(p-1)} \int_{1}^{2} s^{1 /(p-1)} e^{s-2} d s
\end{aligned}
$$

and, as one easily checks, the maximum is equal to 0 . This completes the proof.

Sharpness, $2<p<\infty$. We have, by Young's inequality,

$$
c\|f\|_{p} \leq\|f\|_{p}^{p}+p^{-p /(p-1)}(p-1) c^{p /(p-1)},
$$

so if 1.4 held with some $c<C_{p}$, we would have

$$
\left\|g^{*}\right\|_{1} \leq\|f\|_{p}^{p}+C
$$

for some $C<p^{-p /(p-1)}(p-1) C_{p}^{p /(p-1)}=M_{p}$. Therefore it suffices to show that the smallest $C$ for which 4 is valid equals $M_{p}$.

Suppose, then, that (4.8) holds with some universal $C$, and let us use Theorem 2.3 with $V=V_{p}$ given by $V_{p}(x, y, z)=z-|x|^{p}$. As a result, we obtain a function $U_{0}$ satisfying $1^{\circ}-3^{\circ}$. Observe that for any $(x, y, z) \in \mathcal{A}$ and $t \in \mathbb{R}$

$$
U_{0}(x, y, z)=t+U_{0}(x, y-t, z-t) .
$$

This is a consequence of the fact that the function $V_{p}$ also has this property, and of the very definition of $U_{0}$.

Now it is convenient to split the proof into a few parts.

STEP 1. First we will show that for any $y$,

$$
U_{0}(0, y, y) \geq y+(p-1) p^{-p /(p-1)}=U_{p}(0, y, y) .
$$

In view of 4.9 , it suffices to prove this for $y=0$. Let $d=p^{-1 /(p-1)}$ and $\delta>0$. Applying $1^{\circ \prime}$ to $\varepsilon=-1, x=y=z=0$ and a mean-zero $T$ taking 
values $\delta$ and $-d$, we obtain

$$
U_{0}(0,0,0) \geq \frac{d}{d+\delta} U_{0}(-\delta, \delta, \delta)+\frac{\delta}{d+\delta} U_{0}(d,-d, 0) .
$$

By (4.9), $U_{0}(-\delta, \delta, \delta)=\delta+U_{0}(-\delta, 0,0)$. Furthermore, by $2^{\circ}, U_{0}(d,-d, 0)$ $\geq-d^{p}$, so the above estimate yields

$$
U_{0}(0,0,0) \geq \frac{d}{d+\delta}\left(\delta+U_{0}(-\delta, 0,0)\right)-\frac{\delta}{d+\delta}|d|^{p} .
$$

Similarly, one uses property $1^{\circ \prime}$ and then $2^{\circ}$ to get

$$
\begin{aligned}
U_{0}(-\delta, 0,0) & \geq \frac{d}{d+\delta} U_{0}(0, \delta, \delta)+\frac{\delta}{d+\delta} U_{0}(-d-\delta,-d, 0) \\
& \geq \frac{d}{d+\delta}\left(\delta+U_{0}(0,0,0)\right)-\frac{\delta}{d+\delta}(d+\delta)^{p} .
\end{aligned}
$$

Combining this with (4.11), subtracting $U_{0}(0,0,0)$ from both sides of the resulting estimate, dividing through by $\delta$ and letting $\delta \rightarrow 0$ leads to $U_{0}(0,0,0)$ $\geq d-d^{p}=U_{p}(0,0,0)$, which is what we need.

Consequently, by the definition of $U_{0}$, for any $y \in \mathbb{R}$ and $\kappa>0$ there is a pair $\left(f^{\kappa, y}, g^{\kappa, y}\right) \in M(0, y)$ satisfying

$$
U_{p}(0, y, y) \leq V_{p}\left(f_{\infty}^{\kappa, y}, g_{\infty}^{\kappa, y},\left(g_{\infty}^{\kappa, y}\right)^{*}\right)+\kappa .
$$

STEP 2. Let $N$ be a positive integer and let $\delta=t_{0} / N$, where $t_{0}$ is given by 4.7. We will need the following auxiliary fact.

LEMMA 4.4. There is a universal $R$ such that the following holds. If $x \in\left[\delta, t_{0}\right], y \in \mathbb{R}$ and $T$ is a centered random variable which takes values in $\left[\gamma_{p}\left(G_{p}(x)\right), \delta\right]$, then

$$
\mathbb{E} U_{p}(x-T, y+T,(y+T) \vee y) \leq U_{p}(x, y, y)+R \delta^{2} .
$$

Proof. We start from the observation that for any fixed $x \in\left[\delta, t_{0}\right]$ and $y \in \mathbb{R}$, if $t \in\left[-\gamma_{p}\left(G_{p}(x)\right), 0\right]$,

$$
U_{p}(x-t, y+t, y)=U_{p}(x, y, y)-U_{p x}(x, y, y) t+t .
$$

For $t \in(0, \delta]$, by the concavity of $s \mapsto U_{p}(s, 0,0)$,

$$
\begin{aligned}
U_{p}(x-t, y+t, y+t) & =y+t+U_{p}(x-t, 0,0) \\
& \geq y+t+U_{p}(x, 0,0)-U_{p x}(x, 0,0) t-R \delta^{2} \\
& =U_{p}(x, y, y)-U_{p x}(x, y, y) t+t-R \delta^{2} .
\end{aligned}
$$

Here, for example, one may take $R=-\inf _{x \in\left[0, t_{0}\right]} U_{p x x}(x, 0,0)$, which is finite: see (4.6). The inequality 4.13 follows immediately from the above two estimates.

Now consider a martingale $f=\left(f_{n}\right)_{n=1}^{N}$, starting from $t_{0}$, which satisfies the following condition: if $0 \leq n \leq N-1$, then on the set $\left\{f_{n}=t-n \delta\right\}$, the difference $d f_{n+1}$ takes values $-\delta$ and $-\gamma_{p}\left(G_{p}\left(f_{n}(\omega)\right)\right)$; on the complement of 
this set, $d f_{n+1} \equiv 0$. Let $g$ be the \pm 1 transform of $f$ given by $g_{0}=f_{0}$ and $d g_{n}=-d f_{n}, n=1, \ldots, N$. The key fact about the pair $(f, g)$ is that

$$
\mathbb{E} U_{p}\left(f_{n}, g_{n}, g_{n}^{*}\right) \leq \mathbb{E} U_{p}\left(f_{n+1}, g_{n+1}, g_{n+1}^{*}\right)+R \delta^{2}, \quad n=0,1, \ldots, N-1 .
$$

This is an immediate consequence of Lemma 4.4 (applied conditionally with respect to $\left.\mathcal{F}_{n}\right)$ and the fact that $U_{p}\left(f_{n}, g_{n}, g_{n}^{*}\right) \neq U_{p}\left(f_{n+1}, g_{n+1}, g_{n+1}^{*}\right)$ if and only if $f_{n}=t-n \delta$ or $g_{n}=t+n \delta=g_{n}^{*}$.

The next property of the pair $(f, g)$ is that if $f_{N} \neq 0$, then we have $U_{p}\left(f_{N}, g_{N}, g_{N}^{*}\right)=V_{p}\left(f_{N}, g_{N}, g_{N}^{*}\right)$. Indeed, $f_{N} \neq 0$ implies $d f_{n}>0$ for some $n \geq 1$ and then, by construction,

$$
g_{N}^{*}-g_{N}=g_{n}^{*}-g_{n}=-d g_{n}=d f_{n}=\gamma_{p}\left(f_{n}\right)=\gamma_{p}\left(f_{N}\right) .
$$

Thus we may write

$$
\begin{aligned}
M_{p} & =U_{p}\left(t_{0}, t_{0}, t_{0}\right) \\
& \leq \mathbb{E} U_{p}\left(f_{N}, g_{N}, g_{N}^{*}\right)+R N \delta^{2} \\
& =\mathbb{E} V_{p}\left(f_{N}, g_{N}, g_{N}^{*}\right) 1_{\left\{f_{N} \neq 0\right\}}+U_{p}\left(0,2 t_{0}, 2 t_{0}\right) \mathbb{P}\left(f_{N}=0\right)+R N \delta^{2},
\end{aligned}
$$

since $g_{N}=g_{N}^{*}=2 t_{0}$ on $\left\{f_{N}=0\right\}$.

STEP 3. Now let us extend the pair $(f, g)$ as follows. Fix $\kappa>0$ and put $f_{N}=f_{N+1}=f_{N+2}=\cdots$ and $g_{N}=g_{N+1}=g_{N+2}=\cdots$ on $\left\{f_{N} \neq 0\right\}$, while on $\left\{f_{N}=0\right\}$, let the conditional distribution of $\left(f_{n}, g_{n}\right)_{n \geq N}$ with respect to $\left\{f_{N}=0\right\}$ be that of the pair $\left(f^{\kappa, 2 t_{0}}, g^{\kappa, 2 t_{0}}\right)$, obtained at the end of Step 1 . The process $(f, g)$ we get consists of simple martingales and, by (4.12) and (4.15), we have

$$
M_{p} \leq \mathbb{E} V_{p}\left(f_{\infty}, g_{\infty}, g_{\infty}^{*}\right)+R N \delta^{2}+\kappa \mathbb{P}\left(f_{N}=0\right)
$$

Now it suffices to note that choosing $N$ sufficiently large and $\kappa$ sufficiently small, we can make the expression $R N \delta^{2}+\kappa \mathbb{P}\left(f_{N}=0\right)$ arbitrarily small. This shows that $M_{p}$ is indeed the smallest $C$ which is allowed in 4.8.

Sharpness, $p=\infty$. We may assume that $\|X\|_{\infty}=1$. The proof will be entirely based on the following version of Theorem 2.3 .

TheOREM 4.5. Let $U_{0}:\{(x, y, z):|x| \leq 1, y \leq z\} \rightarrow \mathbb{R}$ be given by

$$
U_{0}(x, y, z)=\mathbb{E} g_{\infty}^{*} \vee z
$$

where the supremum is taken over the class of all pairs $(f, g) \in M(x, y)$ such that $\|f\|_{\infty} \leq 1$. Then $U_{0}$ enjoys the following properties:

$1^{\circ}$ For any $\varepsilon \in\{-1,1\}, x \in[-1,1], y \leq z$ and any simple centered random variable $T$ satisfying $|x+\varepsilon T| \leq 1$, we have

$$
\mathbb{E} U_{0}(x+\varepsilon T, y+T,(y+T) \vee z) \leq U_{0}(x, y, z) .
$$


$2^{\circ} U_{0}(x, y, z) \geq z$ for all $(x, y, z)$ from the domain of $U_{0}$.

$3^{\circ} U_{0}(x, y, y) \leq C_{\infty}$ for all $x, y$ such that $|x|=|y| \in[-1,1]$.

For the proof, modify the argument from [B2]. Note that the function $U_{0}$ satisfies (4.9) (with the obvious restriction to $x$ lying in $[-1,1]$ ).

Now we turn to the optimality of the constant $C_{\infty}$. First we will show that

$$
U_{0}(0,0,0) \geq 1 \text {. }
$$

To prove this, take $\delta \in(0,1)$ and use $1^{\circ}$ to obtain

$$
U_{0}(0,0,0) \geq \frac{1}{1+\delta} U_{0}(\delta, \delta, \delta)+\frac{\delta}{1+\delta} U_{0}(-1,-1,0) .
$$

We have $U_{0}(-1,-1,0) \geq 0$ by $2^{\circ}$, and $U_{0}(\delta, \delta, \delta)=\delta+U(\delta, 0,0)$ by 4.9 . Thus we have

$$
U_{0}(0,0,0) \geq \frac{\delta+U_{0}(\delta, 0,0)}{1+\delta} .
$$

Similarly, using $1^{\circ}$ and then $2^{\circ}$ yields

$$
U(\delta, 0,0) \geq(1-\delta) U_{0}(0, \delta, \delta)+\delta U_{0}(1, \delta-1,0) \geq(1-\delta)\left[\delta+U_{0}(0,0,0)\right] .
$$

Plug this into (4.17), subtract $U_{0}(0,0,0)$ from both sides, divide through by $\delta$ and let $\delta \rightarrow 0$. As a result, one gets (4.16).

Now fix a positive integer $N$ and set $\delta=\left(1-e^{-1}\right) / N$. For any $k=$ $1, \ldots, N$, we have, by $1^{\circ}, 2^{\circ}$ and 4.9 ,

$$
\begin{aligned}
U_{0}(k \delta, 0,0) & \geq \frac{\delta}{1-k \delta+\delta} U_{0}(1, k \delta-1,0)+\frac{1-k \delta}{1-k \delta+\delta} U_{0}((k-1) \delta, \delta, \delta) \\
& \geq \frac{1-k \delta}{1-k \delta+\delta}\left[\delta+U_{0}((k-1) \delta, 0,0)\right],
\end{aligned}
$$

or, equivalently,

$$
\frac{U_{0}(k \delta, 0,0)}{1-k \delta} \geq \frac{U_{0}((k-1) \delta, 0,0)}{1-(k-1) \delta}+\frac{\delta}{1-(k-1) \delta} .
$$

It follows by induction that

$$
e U_{0}\left(1-e^{-1}, 0,0\right)=\frac{U_{0}(N \delta, 0,0)}{1-N \delta} \geq U_{0}(0,0,0)+\sum_{k=1}^{N} \frac{\delta}{1-(k-1) \delta} .
$$

Letting $N \rightarrow \infty$ and using (4.16), we arrive at

$$
e U_{0}\left(1-e^{-1}, 0,0\right) \geq 1+\int_{0}^{1-e^{-1}} \frac{d x}{1-x}=2,
$$

and hence, by 4.9),

$$
U_{0}\left(1-e^{-1}, 1-e^{-1}, 1-e^{-1}\right)=1-e^{-1}+U_{0}\left(1-e^{-1}, 0,0\right) \geq 1+e^{-1} .
$$

It suffices to apply $3^{\circ}$ to complete the proof. 
Acknowledgments. The author would like to thank the referee for comments and suggestions, which improved the presentation of the paper. The research was partially supported by Foundation for Polish Science and MNiSW Grant N N201 397437.

\section{References}

[Bi] K. Bichteler, Stochastic integration and $L^{p}$-theory of semimartingales, Ann. Probab. 9 (1980), 49-89.

[B1] D. L. Burkholder, Explorations in martingale theory and its applications, in: École d'Été de Probabilités de Saint-Flour XIX-1989, Lecture Notes in Math. 1464, Springer, Berlin, 1991, 1-66.

[B2] - Sharp norm comparison of martingale maximal functions and stochastic integrals, in: Proceedings of the Norbert Wiener Centenary Congress, 1994 (East Lansing, MI, 1994), Proc. Sympos. Appl. Math. 52, Amer. Math. Soc., Providence, RI, 1997, 343-358.

[O1] A. Osękowski, Sharp maximal inequality for stochastic integrals, Proc. Amer. Math. Soc. 136 (2008), 2951-2958.

[O2] - Sharp maximal inequality for martingales and stochastic integrals, Electron. Comm. Probab. 14 (2009), 17-30.

[P] G. Peskir, The best Doob-type bounds for the maximum of Brownian paths, Progr. Probab. 43 (1998), 287-296.

Adam Osękowski

Department of Mathematics, Informatics and Mechanics

University of Warsaw

Banacha 2

02-097 Warszawa, Poland

E-mail: ados@mimuw.edu.pl

Received September 3, 2010;

received in final form October 21, 2010 
